\title{
Frequency and Correlation of Common Genes Copy Number Alterations in Childhood Acute Lymphoblastic Leukemia with Prognosis
}

\author{
Abbasali Hosein Pour Feizi ${ }^{1}$, Sirous Zeinali ${ }^{2,3}$, Jacek Toporski ${ }^{4}$, Roghayeh \\ Sheervalilou ${ }^{5}$, Sahar Mehranfar ${ }^{1,6,7 *}$
}

\begin{abstract}
Objective: It was shown by genomic profiling that despite no detectable chromosomal abnormalities a proportion of children with pre-B acute lymphoblastic leukemia harbors copy number alterations (CNA) of genes playing role in B-cell development and function. The aim of the study was to determine the frequency of CNA in pediatric acute lymphoblastic leukemia and correlate these findings with clinical outcome. Methods: DNA extracted from peripheral blood or bone marrow at diagnosis/relapse of fifty newly diagnosed children with precursor B-cell acute lymphoblastic leukemia was analyzed for CNA with multiplex ligation-dependent probe amplification. Results: The analysis revealed 76 CNA in 24 patients most frequently found in PAR1 (17\%), CDKN2A/B (15.7\%) and PAX5 (14.4\%) genes. There were significant CNA co-occurrences between PAX5, CDKN2A/B, BTG1, ETV6, PAR1 or XP22 genes, $(\mathrm{p}<0.020)$ and the high-risk group. There was a significant correlation between $E B F 1, R B 1$, and $I K Z F 1$ alterations and bone marrow relapse. Patients with CNA in screened genes are more likely to succumb to their disease except for those with $P A R 1$ or $X P 22$ genes $(\mathrm{p}<0.050)$. Conclusion: The multiplex ligation-dependent probe amplification could be considered as an independent diagnostic tool allowing prompt identification of patients at high risk of treatment failure and, subsequently, a more adequate treatment approach.
\end{abstract}

Keywords: Childhood acute lymphoblastic leukemia (ALL)- Multiplex ligation-dependent probe amplification (MLPA)

Asian Pac J Cancer Prev, 21 (12), 3493-3500

\section{Introduction}

Acute lymphoblastic leukemia (ALL) is the most common childhood malignancy. About $75 \%$ of children with leukemia have various chromosomal abnormalities. Although $85-90 \%$ of child patients are cured with modern therapies, relapses occur in approximately $15-20 \%$ of cases. Previous studies showed that treatment failure is strongly related to the site and timing of relapse (Bailey et al., 2008; Parker et al., 2010; Moorman, 2012; Yamashita et al., 2013). Also, other studies revealed that the cells existing in the relapse clone, were often present at the time of first diagnosis (Reismüller et al., 2009; Vardiman, 2010). These data suggest that we can determine the risk of relapse by screening common involved genes before chemotherapy and during treatment and also on follow-up (Mullighan et al., 2008a). The risk of specific chromosomal abnormalities that are important for diagnosis and treatment are well-documented (Harrison et al., 2010; Harrison and Johansson, 2015). In about $20-25 \%$ of cases with normal karyotype, further investigation is required to determine the molecular factors affecting the prognosis (Pui et al., 2004; Pieters and Carroll, 2008). Molecular studies have indicated that copy number abnormality (CNAs) of these genes play a central role in the pathobiology of lymphoid leukemia. Many of these alterations have an initiating role in leukemogenesis pathway and they have a significant impact on prognosis and therapeutic decisions (Kuiper et al., 2007; Mullighan et al., 2007; Strefford et al., 2007; Zhou et al., 2012). Previous studies have identified numerous deletions in signaling pathways of precursor B-cell (BCP) differentiation (IKZF1, ETV6, PAX5, and EBF1), cell cycle control, tumor suppression (CDKN2A/2B, BTG1, and RB1), and cytokine receptors

\footnotetext{
${ }^{1}$ Hematology and Oncology Research Center, Tabriz University of Medical Sciences, Tabriz, Iran. ${ }^{2}$ Department of Molecular Medicine, Biotechnology Research Center, Pasteur Institute of Iran, Tehran, Iran. ${ }^{3}$ Kawsar Human Genetics Research Center, Kawsar Genomics Center, Tehran, Iran. ${ }^{4}$ Department of Clinical Sciences, Pediatric Oncology and Hematology, University of Lund, Lund, Sweden. ${ }^{5}$ Pharmacology Research Center, Zahedan University of Medical Sciences, Zahedan, Iran. ${ }^{6}$ Department of Genetics and Immunology, Faculty of Medicine, Urmia University of Medical Sciences, Urmia, Iran. ${ }^{7}$ Social Determinate of Health Research Center, Clinical Research Institute Urmia University of Medical Science, Urmia, Iran. *For Correspondence: dr_sahar21@yahoo.com
} 
(CRLF2, IL3RA, CSF2RA, and SHOX) localized in the pseudoautosomal region (PAR1) (Kuiper et al., 2007; Mullighan et al., 2007).

CNAs of IKZF1 and the most famous genes of PARI region have been shown to be associated with poor prognosis in BCP-ALL (Mullighan et al., 2009b; Russell et al., 2009; Schwab et al., 2010; Yang et al., 2011). Ma et al. in 2015 showed that half of cases of leukemia had multiple sub-clonal mutations in a pathway or genes at diagnosis but mostly with only one minor clone. Their results provide important insights into the genetic basis of chemotherapy failure in ALL (Ma et al., 2015). Single nucleotide polymorphism (SNP), array-based comparative genomic hybridization (aCGH) and fluorescence in situ hybridization (FISH) have been used previously for detection of these abnormalities, but all of them had important disadvantages such as being expensive, labor-intensive and unavailable in most of the labs. Consequently, the aforementioned causes make these methods inapplicable for routine diagnosis or screening large cohorts (Harrison et al., 2005; Coll-Mulet et al., 2008; Russell et al., 2009; Stevens-Kroef et al., 2009; Al Zaabi et al., 2010). Therefore, it is necessary to find a rapid and reliable method for screening these abnormalities to determine their incidence, interrelationship and prognostic significance. Multiplex ligation-dependent probe amplification (MLPA) is a rapid, low-cost and simple multiplex polymerase chain reaction (PCR)-based technique introduced by Schouten in 2002 (Schouten et al., 2002). It can distinguish about 50 different genomic lengths of DNA or RNA sequences in one reaction (www. MRC-Holland.com).

This study has used MLPA method for the simultaneous screening of these abnormalities, applicable to large cohorts of patients with BCP-ALL. A variety of mutations occur more frequently in certain races and ethnic groups, such as CRLF2 in Hispanics (Mullighan et al., 2007; Familiades et al., 2009; Nebral et al., 2009; Harvey et al., 2010). Thus, inherited genetic variations are important in the pathogenesis of ALL (Hunger and Mullighan, 2015). There is no record of CNAs in Iranian ALL patients. Therefore, this study was done to find the prevalence of CNAs in our BCP-ALL patients.

\section{Materials and Methods}

\section{Patient selections}

Patients with BCP-ALL registered with Children's Hospital of Tabriz Medical Sciences University (TBZMED) (2014-2016) were eligible for this study (Table1). They were diagnosed by standard criteria. The inclusion criteria embraced the patients with proven ALL, and being 1-14 years age at the time of first diagnosis or first BM relapse before any salvage therapy. Exclusion criteria embraced the acute leukemia in children with Down syndrome and samples from foreigners. Initially $66 \mathrm{BM}$ and/or peripheral blood (PB) samples were obtained from untreated patients with ALL. All patients were follow up for one year. 21 Patients were categorized as high-risk (HR) or standard risk based on the National Cancer Institute (NCI) criteria. High-risk category included WBC count $\geq 50 \times 109 / \mathrm{ml}$ and/ or age $\geq 10$ years. Standard risk (SR) category included, WBC count $\leq 50 \times 10^{9} / \mathrm{ml}$ and $/$ or age between $1-10$ years old (Mullighan et al., 2009b) (Figure 1).

\section{Sample selection}

DNA samples were obtained freshly from $\mathrm{PB}, \mathrm{BM}$ or both. Additionally, $5 \mathrm{BM}$ and $10 \mathrm{~PB}$ samples from nonmalignant patients matched the samples from control group in terms of age and sex.

\section{DNA extraction}

DNA extraction was done by using the QIAamp DNA minikit (Qiagen, Hilden, Germany). The final DNA concentration was determined by NanoDrop spectrophotometry (NanoDrop, Wilmington, DE, USA) and the purity was assessed by $2 \%$ agarose gel electrophoresis.

\section{Definition of P335-B2 SALSA MLPA kit}

DNA obtained from samples was used to determine the CNAs of IKZF1, CDKN2A/B, PAX5, EBF1, ETV6, BTG1, RB1, and PAR1 region, using the P335-B2 SALSA MLPA kit (MRC-Holland, Amsterdam, the Netherlands) (Schwab et al., 2010). Seven control samples were taken in each run of MLPA, to compare the patients' with control peaks. Relative copy number was gained after normalization of peaks against control. The PCR fragments were separated by capillary electrophoresis on a 3130XL Genetic Analyzer (Life Technologies, Carlsbad, CA, USA). MLPA data were analyzed using GeneMarker V1.95 Soft Genetics (State College, Pennsylvania). The primer sequences for Pax 5 have been presented in supplementary Table 1.

\section{Statistical Analysis}

Normality of the data was assessed by KolmogorovSmirnov test and expressed by the mean and standard deviation. Non-normal variables were implied as median (max-min). The gene CNAs difference between the ALL and the control samples were calculated by independent sample T-test. To assess the agreement between genes detected by MLPA, we used the kappa test. In order to determine the importance of quantitative factors in risk grouping, "independent variable importance" analysis which is a predictive test was employed. Also, to determine the association of genes with risk of relapse and mortality and their predictive importance, we used "contingency coefficient" test. Study variables were compared by the Chi-square or the Mann-Whitney test, for categorical and non-normal numeric variables respectively. P-values $<0.05$ were considered statistically significant. For the kappa test values, the level $>0.7$ were considered strong agreement and $<0.4$ had a weak agreement. All statistical analyses were performed using the Statistical Package for the Social Sciences (SPSS 16.0, Inc, Chicago, IL, USA).

\section{Results}

Sources of DNA samples were from PB in 30 (53.5\%) and $\mathrm{BM}$ in $26(46.5 \%)$ patients. In six patients, both BM and PB samples were taken simultaneously. Molecular 
Table 1. Demographic Data of Acute Lymphoblastic Leukemia Patients

\begin{tabular}{lll}
\hline Demographic data & N (frequency) & \\
\hline Sex n (\%) & Female & $19(38 \%)$ \\
& Male & $31(62 \%)$ \\
Age (years) n (\%) & $1-4$ & $22(44 \%)$ \\
& $5-10$ & $21(42 \%)$ \\
& $10-14$ & $7(14 \%)$ \\
Mean age (mean \pm SD) & Female & $6.0 \pm 2.7$ \\
WBC count n $(\%)$ & Male & $5.8 \pm 3.4$ \\
& $10-49.9$ & $20(40 \%)$ \\
risk group n $(\%)$ & $>50$ & $16(32 \%)$ \\
& Standard risk & $14(28 \%)$ \\
& High-risk & $21(58 \%)$ \\
\hline
\end{tabular}

analysis was carried out on 56 samples from 50 patients. All patients were follow up for one year. 21 patients $(42 \%)$ were assigned to high-risk group and 29 of them $(58 \%)$ to standard risk. 12 patients $(24 \%)$ belonged to $\mathrm{BM}$ relapsed group, 5 of which originally high-risk BCP-ALL. 10 patients (20\%) who died subsequently because of refractory diseases, lodged in mortality group. No significant difference was observed between sex, age and sample source in various groups, except a significant correlation between high $\mathrm{WBC}$ and high-risk group $(\mathrm{p}=0.001)$.

\section{MLPA findings}

Overall, 76 CNAs was found in 24 patients (48\%), who harbored at least one up to eight deletions or duplications in the genes studied. Deletions were more frequent than duplications (59.3\% versus $40.7 \%)$. Also, simultaneous aberrations were observed in different genes. Among all of the aberrations, four patients $(16.6 \%)$ had CNA in one, six $(25 \%)$ had in 2 , nine $(37.5 \%)$ had in $3-4$ and five $(20 \%)$ had in 5-7 genes. One patient, who had CNAs in all the genes under study, subsequently succumbed to refractory multiple relapses. Three most-prevalent CNAs in the order of frequency were XP22 (17\%), CDKN2A/B (15.7\%) and PAX5 (14.4\%), whereas deletion of RB1 (7.8\%) was the least-frequent (Table2).

\section{Relationship between MLPA results with WBC count}

We found a statistically significant relationship between CDKN2A/B and having a high initial WBC count in patients with ALL $(p=0.03)$. Also, there was a considerable, yet not significant relationship between PAX5 and WBC $(p=0.08)$. Three or more deletions were significantly more frequent in adult children $(\mathrm{p}=0.04)$ and in those patients with a high WBC count $(p=0.03)$. Data

I.

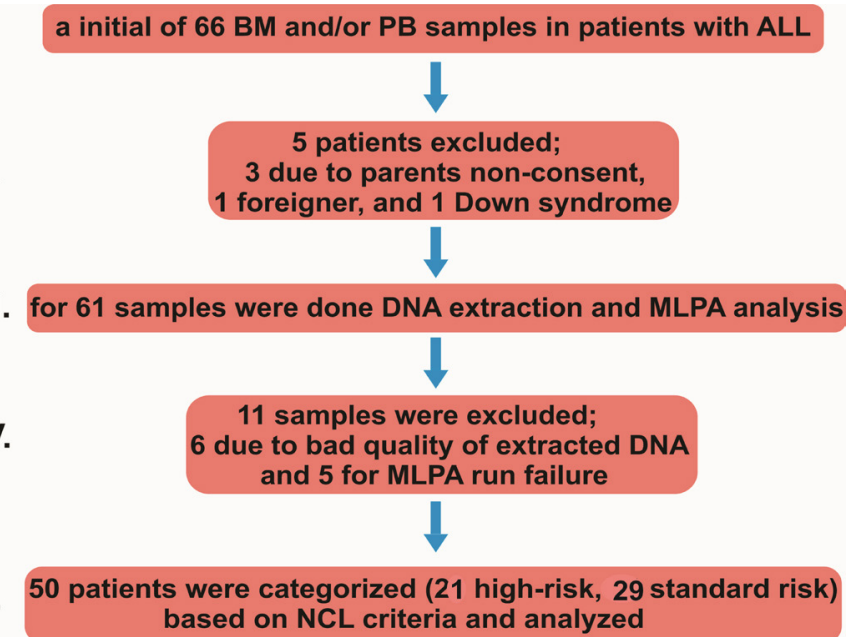

Figure 1. The Sampling Process

Table 2. Prevalence of Gene Copy Number Abnormalities by MLPA Method in Relapse, High-Risk and Mortalitygroups

\begin{tabular}{lccccc}
\hline Gene & $\begin{array}{c}\text { CNA } \\
\text { (frequency) } \\
(\mathrm{n}=76)(\%)\end{array}$ & $\begin{array}{c}\text { High-risk group } \\
(21 \text { pts., } 42 \%) \\
\text { P value* }\end{array}$ & $\begin{array}{c}\text { Relapse Group } \\
(12 \text { pts., } 24 \%) \\
\text { P value* }\end{array}$ & $\begin{array}{c}\text { Mortality group } \\
(10 \text { pts., 20\%) } \\
\text { P value* }\end{array}$ & $\begin{array}{c}\text { Common } \\
\text { involved exons (\%) }\end{array}$ \\
\hline XP22 OR PAR1 & $13(17)$ & 0.002 & 0.325 & 0.325 & Il3RA,P2Ry8,CRLF2 \\
CDKN2A/B & $12(15.7)$ & 0.001 & 0.181 & 0.04 & $5(100), 2 \mathrm{a}(75)$ \\
PAX5 & $11(14.4)$ & 0.002 & 0.463 & 0.048 & $6,7,8(55.5)$ \\
ETV6 & $10(13)$ & 0.002 & 0.1 & 0.048 & $1 \mathrm{a}, 1 \mathrm{~b}(90), 5(62.5)$ \\
IKZF1 & $9(11.8)$ & 0.063 & 0.055 & 0.002 & $1(40), 8(60)$ \\
BTG1 & $8(10)$ & 0.068 & 0.1 & 0.043 & $1(75), 2(50)$ \\
EBF1 & $7(9.2)$ & 0.063 & 0.001 & 0.02 & $10(71), 14(71)$ \\
RB1 & $6(7.8)$ & 0.4 & 0.018 & 0.007 & $26(80)$ \\
\hline
\end{tabular}




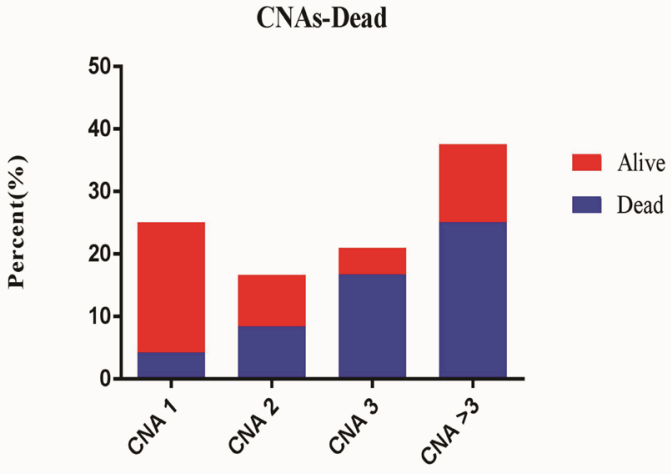

b

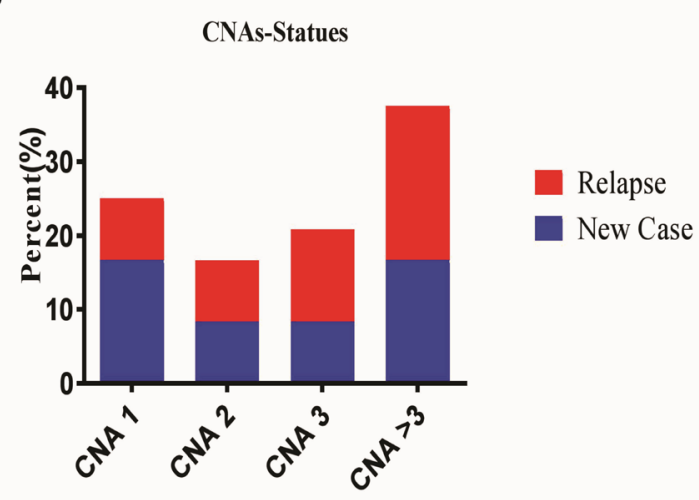

Figure 2. The Evaluation of Correlation between CANs with a) the death in high-risk group, b) the patients relapse. Results showed a significant concurrency between $C N A s$ of $P A X 5, E T V 6, C D K N 2 A / B$ and $P A R 1$ region genes, and being in high-risk group (2a), and a strong correlation between $E B F 1, R B 1$ and $I K Z F 1$ and $B M$ relapse group (2b).

was shown in supplementary Table 2.

Relationship between MLPA findings and risk parameters

We found a significant correlation between $C N A s$ of $P A X 5, C D K N 2 A / B, E T V 6, P A R 1$ region genes (all $\mathrm{p}$-values $<0.020$ ) and being at high-risk group. There was also a strong correlation between $E B F 1, R B 1$, and $I K Z F 1$ ( $\mathrm{p}=0.0001,0.018$ and 0.050 , respectively) and $B M$ relapse group. Patients with CNAs in all interesting genes are more likely to succumb to their disease except $P A R 1$ region genes $(\mathrm{p}$-values $<0.050)$. There was a significant concurrency between CNAs of PAX5, ETV6, CDKN2A/B and $P A R 1$ region genes, and being in high-risk group (Figure 2a). Also, we found strong correlation between $E B F 1, R B 1$ and $I K Z F 1$ and $B M$ relapse group (Figure $2 b)$. Except for $P A R 1$ region genes, patients with all genes $C N A s$ are more likely to succumb to their disease. Patients classified as high-risk group had a greater number of $C N A s$ compared to standard risk group $(\mathrm{p}<0.05)$. CNAs were increasing with age in most of the studied genes. Data was shown in supplementary Table 2 .

The most common involved genes exons

Whole gene deletion in IKZFI was observed in
$40 \%$, of whom $60 \%$ was restricted to exons 1 and 8 . The majority of PAX5 CNAs in our study were in exons 6, 7 and $8(55.5 \%)$. Interestingly, we found a wide range of alteration in the PAX5 gene. Regarding ETV6, the most involved exons were $1 \mathrm{a}, \mathrm{b}$ and exon $5(62.5 \%)$, and in one case all abnormalities were observed. Deletions of $\mathrm{RB} 1$ were the quite rare and they were present only in $8 \%$ of the cases. The most frequent exon was $26(80 \%)$. In addition, deletions within PAR1 region were detected in $17 \%$ of patients. Duplications were more frequent than deletions. The most common duplications were found in IL3RA and P2RY8 genes (Table 2).

\section{Co-occurrence of nine common genes aberrations}

There were novel coincidences between $C N A s$ of the following genes: $C D K N 2 A / 2 B$ and $P A X 5$ (kappa=0.59); $X P 22$ and $E B F 1$ (kappa=0.45); XP22 and $C D K N 2 A / B$ $(\mathrm{kappa}=0.64) ; X P 22$ and $P A X 5$ (kappa=0.59) and $E B F 1$ with ETV6 (kappa $=0.55)$. Also, Strong correlations were observed between ETV6 and BTG1 (kappa=0.8) and RB1 $(\mathrm{kappa}=0.55)$. The positive agreements between genes are presented schematically in Figure 3.

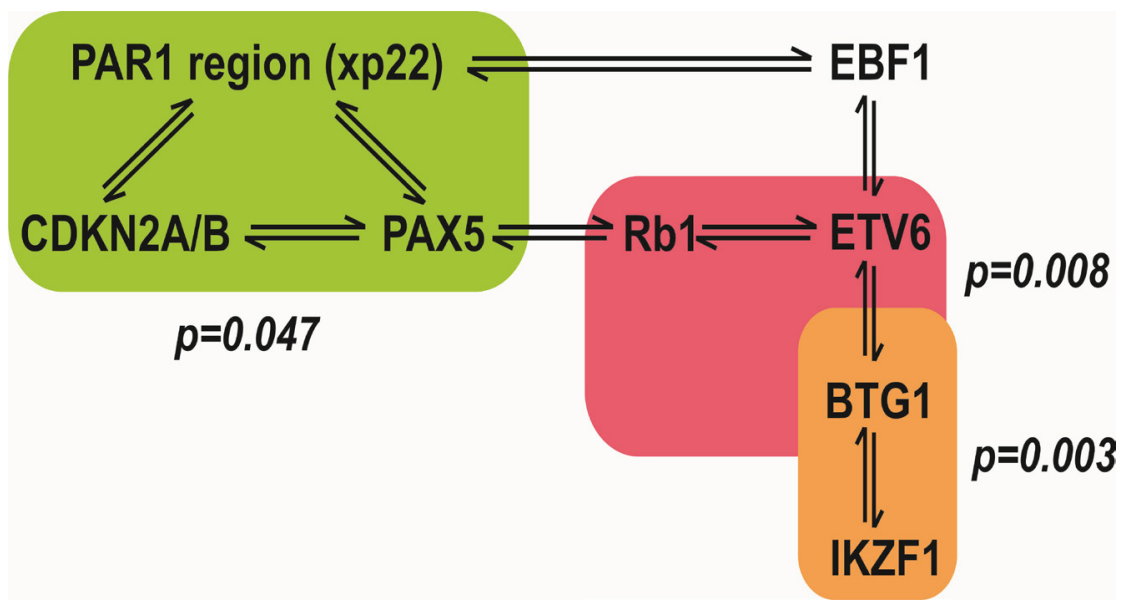

Figure 3. Schematic Correlation between Different Genes under Study Based on Kappa Test Results of Study. Results showed a novel coincidences between $C N A s$ of the following genes: $C D K N 2 A / 2 B$ and $P A X 5 ; X P 22$ and $E B F 1 ; X P 22$ and $C D K N 2 A / B ; X P 22$ and $P A X 5$ and EBF1 with ETV6, and also, strong correlations between ETV6 and BTG1 and $R B 1$. 


\section{Discussion}

We studied the frequency of CNAs of common genes involved in development and differentiation in lymphoid cell (Supplementary Figure. 1) in children with $B C P-A L L$ in Northwest of Iran. In our study the most frequent CNAs were firstly PAR1 region (17\%, 10 Dup and 3 Del). PAR1 region contains at least 24 genes located in pseudoautosomal regions of $\mathrm{X}$ and $\mathrm{Y}$ chromosomes that are an important in hematopoietic development systems (Mullighan et al., 2008b; Mullighan et al., 2009a).

$C N A s$ of $C D K N 2 A / B(15.7 \%, 10 D E L$ and 2DUP $)$ were the second most frequent genes. These tumor suppressors inhibit $C D K 4$ kinase and have the regulatory role in controlling the cell cycle and progression (Martel et al., 1997) and their deletions are important in children with ALL. Mirebeau et al. (2006) demonstrated that patients with $C D K N 2 A / B$ deletions have a shorter time to relapse than others (Yang et al., 2008; Krentz et al., 2013). In line with our results, some studies indicate an adverse effect on risk group (Fizzotti et al., 1995; Carter et al., 2001; Dalle et al., 2002). The prognostic impact of $C D K N 2 A / B$ deletions in pediatric ALL is still controversial (ALLs, 2005; Novara et al., 2009; Sulong et al., 2009; Abdool et al., 2010; Sarhadi et al., 2013).

PAX5 was the third frequent occurring CNAs in our study. Ofverholm et al., (2013) in their study found PAX5 gene alteration second in rank among other genes, having the frequency of about $35 \%$ that is hallmark in relapse of disease, in childhood ALL patients. Our results revealed that this gene had an adverse effect on risk group. PAX5 and $E B F 1$ are transcription factors, which had a key role in STAT5 pathway that is important in differentiation, proliferation and survival (Heltemes-Harris et al., 2011b) and B-cells differentiations (Pongubala et al., 2008; Treiber et al., 2010). We conclude that EBF1 has more important role for determining relapse groups and mortality compared with PAX5 in these patients.

As an anti-proliferative gene, BTG1 affects cell cycle (G0 to G1) and apoptosis. van Galen et al., (2010) concluded that it plays a role in the sensitivity of glucocorticoid receptors, resistance to chemotherapy and increasing the mortality. Similar results were obtained in this study, although in high-risk group, it demonstrated considerable role, yet not significant.

ETV6 is an oncogenic gene. Previous studies found ETV6 deletions in childhood ALL with normal karyotype (Bajaj et al., 2011). There are few studies with fewer subjects, which support the association of ETV6 deletions with poor outcome in adult ALL (Gomez-Segui et al., 2011; Moorman et al., 2012). Our data showed significant correlation between ETV6 and increase in risk of disease and mortality.

$I K Z F 1$ is a tumor suppressor gene and a transcription factor regulator of lymphocyte. The isoforms of $I K Z F 1$ differ in the number of N-terminal zinc finger motifs that bind DNA and are thought to function as dominant-negative factors. Deletions of exons 4-7 result in expression of dominant Negative IKZF1 isoform and shows oncogenic activity (Iacobucci et al., 2008). Loss of exon 8 will have an effect on dimerization of IKZF1, and results in inactivation, therefore it has the same impact as whole gene deletions (Mullighan et al., 2008a). Heltemes et al. showed that $I K Z F$ land $P A X 5$ were the most common mutations in their ALL patients (Heltemes-Harris et al., 2011a). In our study, deletion in exon $8(60 \%)$ of IKZFI is common.

Our data show that association between the number of CNAs and higher initial WBC is more important than age range in all of interesting genes. Moreover, about $75 \%$ of the patients had concurrent CNAs in interesting genes. The co-occurrence of $C D K N 2 A / B$ and $P A X 5$ genes in the mortality and high risk group were high $6(25 \%)$, as illustrated by the previous studies conducted by others (Yang et al., 2008; Mullighan et al., 2009b; van der Veer et al., 2013). These patients often died before achieving remission with induction or re-induction chemotherapy. Besides, we showed the importance of co-occurrence between $P A X 5$ or $C D K N 2 A / 2 B$ and $P A R 1$ region and between ETV6, BTG1 and IKZF1. The clinical impact of alterations in RB1, BTG1 and ETV6 genes in childhood ALL have not yet been fully appreciated by other researchers (Moorman et al., 2010; Moorman et al., 2012; Schwab et al., 2013).

Similar to previous studies, our study also demonstrated that patients without any $C N A s$, were found in a standard risk group with better outcomes (Moorman et al., 2014; Barbosa et al., 2015). These finding indicate that each of these genes not only have a unique unfavorable effect on the disease process, but also may have a worse synergic effect on prognosis. We can hypothesize that their synergy effect on risk of disease and death are related to their linkage to the same chromosome (i.e.; PAX5 and $C D K N 2 A / B$ on chromosome 9). Indeed, Concurrent deletion of $P A X 5$ and $C D K N 2 A / 2 B$ suggests that there are common targets in the pathogenesis of $B C P-A L L$ (Kim et al., 2011; Schwab et al., 2013). One of the first antigens expressed on pro-B cells before transforming to blast is $C D 19$, which is expressed by $P A X 5$. On the other hand, the function of each gene could be modified by other genes in differentiation of blast cells.

These findings highlight the importance of identifying common genes in each population and their screening for identification of high risk patients. Our results are similar to other studies such as the findings of aforementioned studies but, there were some differences due to ethnic origin (Mullighan et al., 2007; Mullighan et al., 2008b; Moorman et al., 2012; Bhandari et al., 2017; Gupta et al., 2017).

It is obvious that increasing the frequency of mutations and involving larger loci of each gene have stronger effect on risk of disease and mortality. The correlation between these genes is important for decision on treatment plan and prediction of ALL prognosis (Gupta et al., 2017). Our results are in line with the findings of the comprehensive study conducted by Mullighan et al., (2008). However, there were some differences due to ethnic origin.

This study demonstrated that the pattern of CNAs in following genes, are different. In addition, there is an association between CNAs of these genes and the risk of being grouped in the risk group. Differences in results in our population compared with other population can be 
explained by ethnic factors. Finally, our perception of the genetic mechanisms of relapse has improved significantly in the last few years, yet using this genetic knowledge and applying this information in clinical practices remain as important challenges. In addition, our results showed that MLPA is reliable method, appropriate for screening especially in countries with high prevalence of cancer and low income. However, due to the low number of patients, a study with a large number of patients is required to confirm or reject the results of this study.

\section{Acknowledgements}

The authors thank all the patients and their parents participated in study. Also, many thanks of the Department of Molecular Medicine, Faculty of Advanced Medical Science of Tabriz University for their support. We would like to thank Mohammad Asghari Jafarabadi for statistical analysis of data of study. We appreciate Abolfazl Barzegari for all his help in this study.

\section{Ethical Approval}

"All procedures performed in studies involving human participants were in accordance with the ethical standards of the institutional and/or national research committee and with the 1964 Helsinki declaration and its later amendments or comparable ethical standards". The study protocol was approved by the ethics of committee of Tabriz University of Medical Sciences.

"Informed consent was obtained from all individual participants included in the study."

\section{Funding Statement}

This study was supported by or Hematology and Oncology Research Center of Tabriz University of Medical Sciences. This project was part of $\mathrm{PhD}$ thesis (study ID: 93/4-10/4) of Sahar Mehranafar.

\section{References}

Abdool A, Donahue AC, Wohlgemuth JG, et al (2010). Detection, analysis and clinical validation of chromosomal aberrations by multiplex ligation-dependent probe amplification in chronic leukemia. PLoS One, 5, e15407.

Al Zaabi EA, Fernandez LA, Sadek IA, et al (2010). Multiplex ligation-dependent probe amplification versus multiprobe fluorescence in situ hybridization to detect genomic aberrations in chronic lymphocytic leukemia: a tertiary center experience. J Mol Diagn, 12, 197-203.

ALLs B-1 (2005). CDKN2 deletions have no prognostic value in childhood precursor-B acute lymphoblastic leukaemia. Leukemia, 1, 4.

Bailey LC, Lange BJ, Rheingold SR, et al (2008). Bone-marrow relapse in paediatric acute lymphoblastic leukaemia. Lancet Oncol, 9, 873-83.

Bajaj R, Xu F, Xiang B, et al (2011). Evidence-based genomic diagnosis characterized chromosomal and cryptic imbalances in 30 elderly patients with myelodysplastic syndrome and acute myeloid leukemia. Mol Cytogenetics, 4, 1.

Barbosa TC, Terra-Granado E, Magalhães IMQ, et al (2015). Frequency of copy number abnormalities in common genes associated with B-cell precursor acute lymphoblastic leukemia cytogenetic subtypes in Brazilian children. Cancer
Genet, 208, 492-501.

Bhandari P, Ahmad F, Das BR (2017). Molecular profiling of gene copy number abnormalities in key regulatory genes in high-risk B-lineage acute lymphoblastic leukemia: frequency and their association with clinicopathological findings in Indian patients. Med Oncol, 34, 92.

Carter TL, Watt PM, Kumar R, et al (2001). Hemizygous p16 INK4A deletion in pediatric acute lymphoblastic leukemia predicts independent risk of relapse. Blood, 97, 572-4.

Coll-Mulet L, Santidrián AF, Cosialls AM, et al (2008). Multiplex ligation-dependent probe amplification for detection of genomic alterations in chronic lymphocytic leukaemia. Br J Haematol, 142, 793-801.

Dalle JH, Fournier M, Nelken B, et al (2002). p16INK4a immunocytochemical analysis is an independent prognostic factor in childhood acute lymphoblastic leukemia. Blood, 99, 2620-3.

Familiades J, Bousquet M, Lafage-Pochitaloff M, et al (2009). PAX5 mutations occur frequently in adult B-cell progenitor acute lymphoblastic leukemia and PAX5 haploinsufficiency is associated with BCR-ABL1 and TCF3-PBX1 fusion genes: a GRAALL study. Leukemia, 23, 1989-98.

Fizzotti M, Cimino G, Pisegna S, et al (1995). Detection of homozygous deletions of the cyclin-dependent kinase 4 inhibitor (p16) gene in acute lymphoblastic leukemia and association with adverse prognostic features. Blood, $\mathbf{8 5}$, 2685-90.

Gomez-Segui I, Such E, Cervera J, et al Gene microdeletions in adult and pediatric acute lymphoblastic leukemia. Blood, 2011. AMER SOC HEMATOLOGY 1900 M STREET. NW SUITE 200, WASHINGTON, DC 20036 USA, 1510.

Gupta SK, Bakhshi S, Kumar L, et al (2017). Gene copy number alteration profile and its clinical correlation in B-cell acute lymphoblastic leukemia. Leuk Lymphoma, 58, 333-42.

Harrison CJ, Haas O, Harbott J, et al (2010). Detection of prognostically relevant genetic abnormalities in childhood B-cell precursor acute lymphoblastic leukaemia: recommendations from the Biology and Diagnosis Committee of the International Berlin-Frankfürt-Münster study group. Br J Haematol, 151, 132-42.

Harrison CJ, Johansson B (2015). Acute lymphoblastic leukemia. Cancer Cytogenetics: Chromosomal and Molecular Genetic Aberrations of Tumor Cells, 198.

Harrison CJ, Moorman AV, Barber KE, et al (2005). Interphase molecular cytogenetic screening for chromosomal abnormalities of prognostic significance in childhood acute lymphoblastic leukaemia: a UK Cancer Cytogenetics Group Study. Br J Haematol, 129, 520-30.

Harvey RC, Mullighan CG, Chen I-M, et al (2010). Rearrangement of CRLF2 is associated with mutation of JAK kinases, alteration of IKZF1, Hispanic/Latino ethnicity, and a poor outcome in pediatric B-progenitor acute lymphoblastic leukemia. Blood, 115, 5312-21.

Heltemes-Harris LM, Willette MJ, Ramsey LB, et al (2011a). Ebf1 or Pax5 haploinsufficiency synergizes with STAT5 activation to initiate acute lymphoblastic leukemia. $J$ Exp Med, 208, 1135-49.

Heltemes-Harris LM, Willette MJ, Ramsey LB, et al (2011b). Ebf1 or Pax5 haploinsufficiency synergizes with STAT5 activation to initiate acute lymphoblastic leukemia. $J$ Exp Med, 20101947.

Hunger SP, Mullighan CG (2015). Acute lymphoblastic leukemia in children. $N$ Engl J Med, 373, 1541-52.

Iacobucci I, Lonetti A, Messa F, et al (2008). Expression of spliced oncogenic Ikaros isoforms in Philadelphia-positive acute lymphoblastic leukemia patients treated with tyrosine kinase inhibitors: implications for a new mechanism of 
resistance. Blood, 112, 3847-55.

Kim M, Choi JE, She CJ, et al (2011). PAX5 deletion is common and concurrently occurs with CDKN2A deletion in B-lineage acute lymphoblastic leukemia. Blood Cells Mol Dis, 47, 62-6.

Krentz S, Hof J, Mendioroz A, et al (2013). Prognostic value of genetic alterations in children with first bone marrow relapse of childhood B-cell precursor acute lymphoblastic leukemia. Leukemia, 27, 295-304.

Kuiper R, Schoenmakers E, Van Reijmersdal S, et al (2007). High-resolution genomic profiling of childhood ALL reveals novel recurrent genetic lesions affecting pathways involved in lymphocyte differentiation and cell cycle progression. Leukemia, 21, 1258-66.

Ma X, Edmonson M, Yergeau D, et al (2015). Rise and fall of subclones from diagnosis to relapse in pediatric B-acute lymphoblastic leukaemia. Nat Commun, 6.

Martel V, Guerci A, Humbert JC, et al (1997). De novo methylation of tumour suppressor genes CDKN2A and CDKN2B is a rare finding in B-cell chronic lymphocytic leukaemia. Br J Haematol, 99, 320-4.

Moorman AV (2012). The clinical relevance of chromosomal and genomic abnormalities in B-cell precursor acute lymphoblastic leukaemia. Blood Rev, 26, 123-35.

Moorman AV, Enshaei A, Schwab C, et al (2014). A novel integrated cytogenetic and genomic classification refines risk stratification in pediatric acute lymphoblastic leukemia (ALL). Blood, blood-2014-03-562918.

Moorman AV, Ensor HM, Richards SM, et al (2010). Prognostic effect of chromosomal abnormalities in childhood B-cell precursor acute lymphoblastic leukaemia: results from the UK Medical Research Council ALL97/99 randomised trial. Lancet Oncol, 11, 429-38.

Moorman AV, Schwab C, Ensor HM, et al (2012). IGH@ translocations, CRLF2 deregulation, and microdeletions in adolescents and adults with acute lymphoblastic leukemia. J Clin Oncol, 2011.40. 3907.

Mullighan CG, Collins-Underwood JR, Phillips LA, et al (2009a). Rearrangement of CRLF2 in B-progenitor-and Down syndrome-associated acute lymphoblastic leukemia. Nat Genet, 41, 1243-6.

Mullighan CG, Goorha S, Radtke I, et al (2007). Genomewide analysis of genetic alterations in acute lymphoblastic leukaemia. Nature, 446, 758-64.

Mullighan CG, Miller CB, Radtke I, et al (2008a). BCR-ABL1 lymphoblastic leukaemia is characterized by the deletion of Ikaros. Nature, 453, 110-4.

Mullighan CG, Phillips LA, Su X, et al (2008b). Genomic analysis of the clonal origins of relapsed acute lymphoblastic leukemia. Science, 322, 1377-80.

Mullighan CG, Su X, Zhang J, et al (2009b). Deletion of IKZF1 and prognosis in acute lymphoblastic leukemia. $N$ Engl J Med, 360, 470-80.

Nebral K, Denk D, Attarbaschi A, et al (2009). Incidence and diversity of PAX5 fusion genes in childhood acute lymphoblastic leukemia. Leukemia, 23, 134-43.

Novara F, Beri S, Bernardo ME, et al (2009). Different molecular mechanisms causing 9p21 deletions in acute lymphoblastic leukemia of childhood. Hum Genet, 126, 511-20.

Öfverholm I, Tran A, Heyman M, et al (2013). Impact of IKZF1 deletions and PAX5 amplifications in pediatric B-cell precursor ALL treated according to NOPHO protocols. Leukemia, 27, 1936.

Parker C, Waters R, Leighton C, et al (2010). Effect of mitoxantrone on outcome of children with first relapse of acute lymphoblastic leukaemia (ALL R3): an open-label randomised trial. The Lancet, 376, 2009-17.
Pieters R, Carroll WL (2008). Biology and treatment of acute lymphoblastic leukemia. Pediatric Clin North Am, 55, 1-20.

Pongubala JM, Northrup DL, Lancki DW, et al (2008). Transcription factor EBF restricts alternative lineage options and promotes B cell fate commitment independently of Pax5. Nat Immunol, 9, 203-15.

Pui C-H, Relling MV, Downing JR (2004). Acute lymphoblastic leukemia. $N$ Engl J Med, 350, 1535-48.

Reismüller B, Attarbaschi A, Peters C, et al (2009). Long-term outcome of initially homogenously treated and relapsed childhood acute lymphoblastic leukaemia in Austria-A population-based report of the Austrian Berlin-FrankfurtMünster (BFM) Study Group. Br J Haematol, 144, 559-70.

Russell LJ, Capasso M, Vater I, et al (2009). Deregulated expression of cytokine receptor gene, CRLF2, is involved in lymphoid transformation in B-cell precursor acute lymphoblastic leukemia. Blood, 114, 2688-98.

Sarhadi VK, Lahti L, Scheinin I, et al (2013). Targeted resequencing of $9 p$ in acute lymphoblastic leukemia yields concordant results with array $\mathrm{CGH}$ and reveals novel genomic alterations. Genomics, 102, 182-8.

Schouten JP, McElgunn CJ, Waaijer R, et al (2002). Relative quantification of 40 nucleic acid sequences by multiplex ligation-dependent probe amplification. Nucleic Acids Res, 30, e57-e.

Schwab C, Jones L, Morrison H, et al (2010). Evaluation of multiplex ligation-dependent probe amplification as a method for the detection of copy number abnormalities in B-cell precursor acute lymphoblastic leukemia. Genes, Chromosomes Cancer, 49, 1104-13.

Schwab CJ, Chilton L, Morrison H, et al (2013). Genes commonly deleted in childhood B-cell precursor acute lymphoblastic leukemia: association with cytogenetics and clinical features. Haematologica Haematol, 2013, 085175.

Stevens-Kroef M, Simons A, Gorissen H, et al (2009). Identification of chromosomal abnormalities relevant to prognosis in chronic lymphocytic leukemia using multiplex ligation-dependent probe amplification. Cancer Genet Cytogenetics, 195, 97-104.

Strefford J, Worley H, Barber K, et al (2007). Genome complexity in acute lymphoblastic leukemia is revealed by array-based comparative genomic hybridization. Oncogene, 26, 4306-18.

Sulong S, Moorman AV, Irving JA, et al (2009). A comprehensive analysis of the CDKN2A gene in childhood acute lymphoblastic leukemia reveals genomic deletion, copy number neutral loss of heterozygosity, and association with specific cytogenetic subgroups. Blood, 113, 100-7.

Treiber T, Mandel EM, Pott S, et al (2010). Early B cell factor 1 regulates $\mathrm{B}$ cell gene networks by activation, repression, and transcription-independent poising of chromatin. Immunity, 32, 714-25.

van der Veer A, Waanders E, Pieters R, et al (2013). Independent prognostic value of BCR-ABL1-like signature and IKZF1 deletion, but not high CRLF2 expression, in children with B-cell precursor ALL. Blood, 122, 2622-9.

van Galen JC, Kuiper RP, van Emst L, et al (2010). BTG1 regulates glucocorticoid receptor autoinduction in acute lymphoblastic leukemia. Blood, 115, 4810-9.

Vardiman JW (2010). The World Health Organization (WHO) classification of tumors of the hematopoietic and lymphoid tissues: an overview with emphasis on the myeloid neoplasms. Chemico Biol Interactions, 184, 16-20.

Yamashita Y, Shimada A, Yamada T, et al (2013). IKZF1 and CRLF2 gene alterations correlate with poor prognosis in Japanese BCR-ABL1-negative high-risk B-cell precursor acute lymphoblastic leukemia. Pediatric Blood Cancer, 
60, 1587-92.

Yang JJ, Bhojwani D, Yang W, et al (2008). Genome-wide copy number profiling reveals molecular evolution from diagnosis to relapse in childhood acute lymphoblastic leukemia. Blood, 112, 4178-83.

Yang YL, Hung CC, Chen JS, et al (2011). IKZF1 deletions predict a poor prognosis in children with B-cell progenitor acute lymphoblastic leukemia: A multicenter analysis in Taiwan. Cancer Sci, 102, 1874-81.

Zhou Y, You MJ, Young KH, et al (2012). Advances in the molecular pathobiology of B-lymphoblastic leukemia. Hum Pathol, 43, 1347-62.

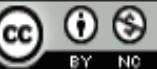

This work is licensed under a Creative Commons AttributionNon Commercial 4.0 International License. 